\title{
HEAT BALANCE RESEARCH OF 3MW MARINE WIND TURBINE'S ENGINE ROOM
}

\author{
NA KANG, YANLONG JIANG, NIANYONG ZHOU, HONG SHI, ZHILI LIU AND YU WANG \\ Man-Machine-Environment Engineering, College of Aerospace Engineering, \\ Nanjing University of Aeronautics and Astronautics, Yudao Street, Nanjing 210016, Jiangsu, China \\ kangnaconnie@yahoo.cn
}

\begin{abstract}
The increment of heat load in Wind Turbine's engine room could cause the system shut down. In this paper the heat balance of Wind Turbine's engine room is investigated according to the structure of the engine room, environmental operating requirements as well as heat-dissipating of different components of a 3MW Wind Turbine System. The heat load of the engine room is analyzed and the physical model is developed based on finite volume method. After that, the temperature distribution of the cabin is obtained under different conditions with/without the jet unit. The temperature distribution in engine compartments with different number and the arrangement of jet units is compared in this paper. Based on the analysis, the cabin cooling design is carried out to achieve the purpose of stopping heat gathering and keeping heat balance of the cabin. This work puts forward the heat balance maintenance and completes the corresponding research, which could be helpful for the optimization of large-capacity Wind Turbine cooling system.
\end{abstract}

Keywords: Wind turbine; heat balance; finite volume method.

\section{Introduction}

Wind power is a kind of renewable and environmental-friendly energy source in the nature with attractive application for providing electric power. Wind turbine power generation, as a mature technology, plays a significant role in the field of wind power utilization. Cooling system is a key issue for the design of wind turbine generator since the cooling performance has an important effect on the generation capacity as well as stability and security of the unit ${ }^{1}$. The hermetical engine room, where the power generator, converter and transformer are held together, prevents the main components from corrosion of salt spray and rain $^{2-4}$. Due to different controlling temperature of the engine room and components, some heat created by components such as power generator, converter and transformer would be inevitably transmitted into the engine room in spite of some relevant cooling methods. As a result, the induced heat load increasing would directly cause the wind turbine shut down ${ }^{5}$. Therefore heat balance analysis of the engine room should be carried out. Only insufficient test data has been obtained from certain conventional experiments on heat transfer of the components in the cabin due to the 
limited equipments and testing methods. In addition, it is difficult to carry out experimental research considering the compactness and asymmetry of the cabin structure. Recently, the numerical methods have achieved great progress, which could significantly reduce the experimental cost. However, the establishment of mathematic model of the temperature field and flow field of the cabin as well as the solution of partial differential equation set would lead to the inconvenience of conversion and collation. Considering the above factors, the commercial CFD (Computational Fluid Dynamics) methods are adopted to conduct the numerical simulation of temperature distribution in the cabin ${ }^{6,7}$.

Jet unit technology which injects cold air into the cabin is applied to adjust the cabin temperature distribution ${ }^{8,9}$. The arrangement of jet units is a critical factor for the heat balance of the cabin. In this paper, a physical model of engine room of a $3 \mathrm{MW}$ marine wind turbine is proposed and the finite volume method is used to simulate the air flow and heat transfer in cabin. The effect of number and arrangements of jet units on the simulation results is investigated. The achievement of analysis could provide reference for optimization of large-capacity wind turbine cooling system.

\section{Mathematical and Physical Model}

\subsection{Mathematical model}

The mathematical model of the cabin is established based on the three-dimensional finite volume method. The air flow in the cabin is considered to be steady, transiently turbulent and compressible. Meanwhile, the convection and thermal radiation between solid walls caused by gravity is ignored. The coupling process of air flow and heat transfer in the cabin can be described by the following equations and calculations ${ }^{10,11}$ :

(i) Mass conservation equation

$$
\frac{\partial}{\partial x_{i}}\left(\rho u_{i}\right)=0 \quad(i=1,2,3),
$$

(ii) Momentum conservation equation

$$
\frac{\partial}{\partial x_{i}}\left(\rho u_{i} u_{j}\right)=\frac{\partial}{\partial x_{i}}\left[\left(\mu+\mu_{t}\right) \frac{\partial u_{j}}{\partial x_{i}}\right]+\frac{\partial}{\partial x_{i}}\left[\left(\mu+\mu_{t}\right) \frac{\partial u_{i}}{\partial x_{j}}\right]-\frac{\partial}{\partial x_{i}}\left(p+\frac{2}{3} \rho k\right),
$$

(iii) Energy conservation equation

$$
\frac{\partial}{\partial x_{i}}\left(\rho u_{i} T\right)=\frac{\partial}{\partial x_{i}}\left[\left(\frac{\mu}{\operatorname{Pr}}+\frac{\mu_{t}}{\sigma}\right) \frac{\partial T}{\partial x_{i}}\right],
$$

(iv) Turbulent kinetic energy equation 


$$
\frac{\partial}{\partial x_{i}}\left(\rho k u_{i}\right)=\frac{\partial}{\partial x_{i}}\left[\left(\mu+\frac{\mu_{t}}{\sigma_{k}}\right) \frac{\partial k}{\partial x_{i}}\right]+G_{k}-\rho \varepsilon,
$$

(v) Turbulent kinetic energy dissipation rate equation

$$
\frac{\partial}{\partial x_{i}}\left(\rho \varepsilon u_{i}\right)=\frac{\partial}{\partial x_{i}}\left[\left(\mu+\frac{\mu_{t}}{\sigma_{\varepsilon}}\right) \frac{\partial \varepsilon}{\partial x_{i}}\right]+C_{1 \varepsilon} \frac{\varepsilon}{k} G_{k}-C_{2 \varepsilon} \rho \frac{\varepsilon^{2}}{k},
$$

where $u$ is velocity, $\mu$ is kinetic viscosity, $\mu_{t}$ is turbulent kinetic viscosity, $p$ is pressure, $\rho$ is density, $T$ is temperature, $x_{i}$ are space coordinates with $i, j$ indicating their directions, $\operatorname{Pr}$ is prandtl number, $k$ is turbulent kinetic energy, $\varepsilon$ is turbulent kinetic energy dissipation rate, $\sigma_{k}$ and $\sigma_{\varepsilon}$ are turbulent prandtl numbers of $k$ and $\varepsilon$ respectively, $C_{1 \varepsilon}$ and $C_{2 \varepsilon}$ are constants, and $G_{k}$ is the production term of turbulent kinetic energy $k$ caused by the velocity gradient.

\subsection{Physical model}

Heat load of the cabin is mainly caused by heat dissipation of internal components, outside solar radiation as well as heat and mass transfer outside and inside the cabin. The heat load of the cabin under the toughest conditions should be calculated and appropriate cooling measures should be taken to control the cabin temperature and heat distribution. The schema of the arrangement of the engine room of a $3 \mathrm{MW}$ offshore wind turbine is shown in Figure 1.

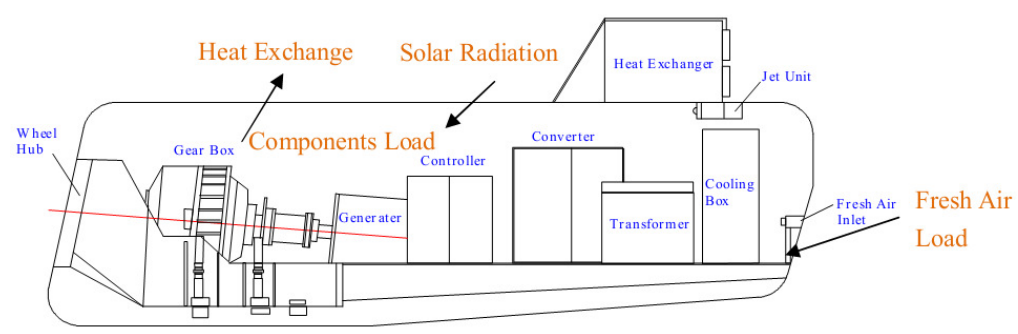

Fig. 1. Schema of the engine room of a $3 \mathrm{MW}$ offshore wind turbine.

As shown in the figure, the internal heat source in the cabin comes from the gear box, generator, control cabinet, inverter, transformer and jet unit arranged. Meanwhile, the external heat load is composed by solar radiation, fresh air system for positive pressure maintenance and heat transfer through the outside wall. 


\section{Model Calculation and Analysis}

\subsection{Model and load analysis}

Three-dimensional solid model of the cabin is established according to the actual size of the engine room. Components with small size or having little effect on airflow and heat transfer are simplified or neglected. Figure 2 shows the 3-D image of the cabin.

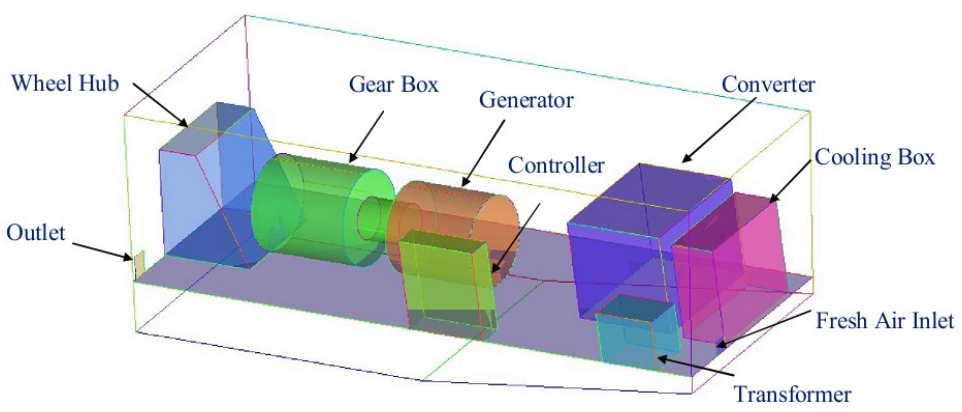

Fig. 2. Domain of calculation model of the engine room.

Heat load of components in the engine room are listed in table 1.

Table 1. Heat load of components in the engine room.

\begin{tabular}{ccccccc}
\hline Component name & gear box & inverter & generator & transformer & cooling box & control cabinet \\
\hline Heat transfer area $\left(\mathrm{m}^{2}\right)$ & 16 & 27.5 & 8.8 & 5.2 & 15.7 & 9.2 \\
Heat dissipating capacity $(\mathrm{W})$ & 5935 & 5635 & 1321 & 3000 & 23600 & 500 \\
Controlling temperature ( $\left.{ }^{\circ} \mathrm{C}\right)$ & 75 & 60 & 60 & 60 & 50 & 50 \\
Proportion of the total heat $(\%)$ & 14.8 & 14.1 & 3.3 & 7.5 & 59.0 & 1.3 \\
\hline
\end{tabular}

\subsection{Boundary conditions}

Firstly, the viscous heating effects are assumed to be negligible and the fluid properties are considered as constant. The inlet boundary region is set as velocity inlet with an initial velocity of $5 \mathrm{~m} / \mathrm{s}$ and an initial temperature of $35{ }^{\circ} \mathrm{C}$. For the outlet boundary, the pressure outlet is used with a zero outlet boundary temperature gradient. In the cabin, the atmospheric pressure is $1.01 \times 10^{5} \mathrm{~Pa}$. The left boundary condition for the wall is naturalized and wall heat transfer coefficient is set as known. For each component, surface temperature or heat generation rate is chosen as the boundary conditions. Besides, the solar radiation is taken into consideration as the extremely hot weather in summer. 


\subsection{Mesh generation}

The huge size and complexity of the engine room cause many difficulties in mesh generation and calculation. Therefore, in this paper the unstructured hexahedral mesh is applied and for some key parts mesh would be refined. A grid independence test for the case is conducted to ensure the accuracy of the numerical results and to determine an appropriate grid density. A reasonable grid number with 600000 cells is selected to yield fairly good results in this paper.

\subsection{Results and analysis}

\section{(1) Heat transfer coefficient of the cabin}

Wind turbine generator is cooled by air with a relatively high velocity which usually reaches $10 \sim 20 \mathrm{~m} / \mathrm{s}$. Due to the asymmetric structure and flow disturbance of the blades, the velocity field is rather uneven. Consequently, the numerical methods are applied to obtain the velocity field and heat transfer coefficient distribution of the cabin.

Figure 3 gives the simulation results of wind field around the cabin; Figure 4 gives convection heat transfer coefficient ahead of and behind the engine room and Figure 5 gives transfer coefficient above and below the engine room when external wind speed is $10 \mathrm{~m} / \mathrm{s}$.

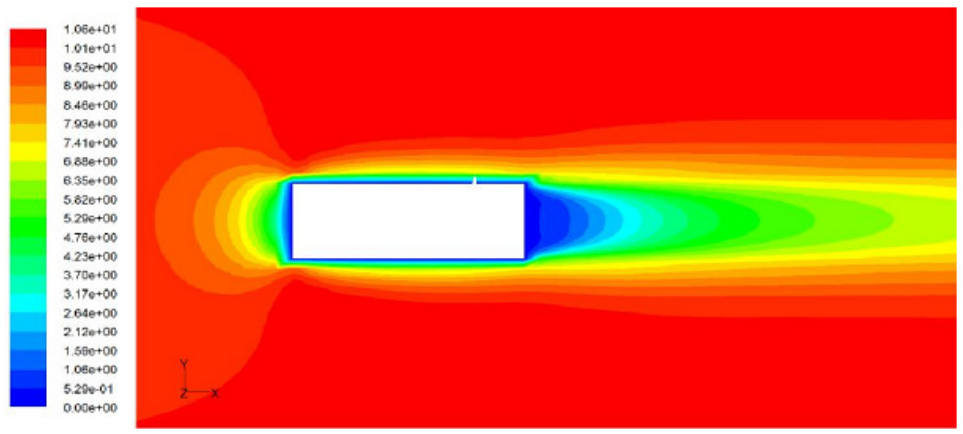

Fig. 3. Wind velocity distribution outside the engine room.

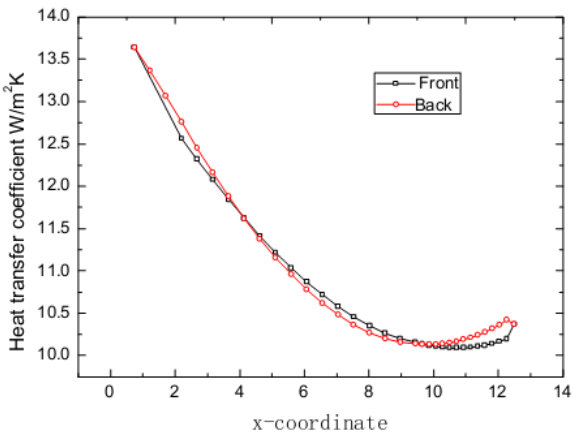

Fig. 4. Heat transfer coefficient of section $\mathrm{z}=5000 \mathrm{~m}$.

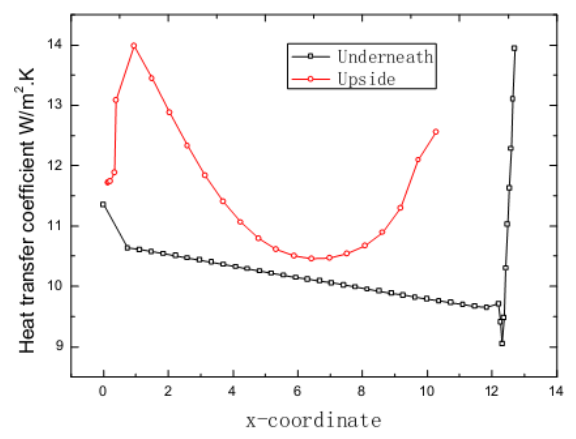

Fig. 5. Heat transfer coefficient of section $y=1500 \mathrm{~mm}$. 
From Figure 4 to 5, we can see that surface convection heat transfer coefficient of the cabin is not constant. Its numerical values fluctuate narrowly between $10 \sim 14 \mathrm{~W} / \mathrm{m}^{2} \mathrm{~K}$. To calculate the temperature field inside the cabin, this heat transfer coefficient is set as an input parameter as a boundary condition.

(2) Temperature field calculation

Figure 6 shows temperature contours within the cabin. Here, the heat dissipation of internal components, outside solar radiation, influx of fresh air as well as heat and mass transfer outside and inside the cabin without jet unit are considered.

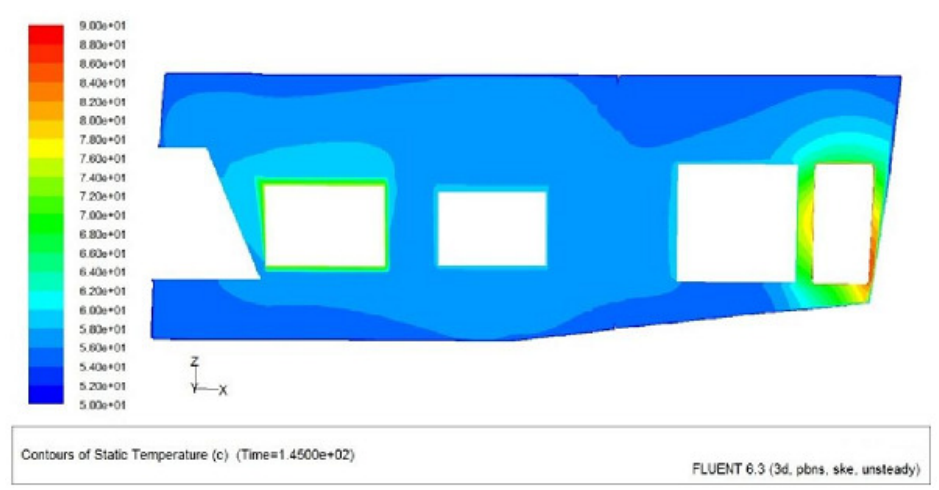

Fig. 6(A). Temperature distribution of the cabin without jet unit of section $y=1500 \mathrm{~mm}$.

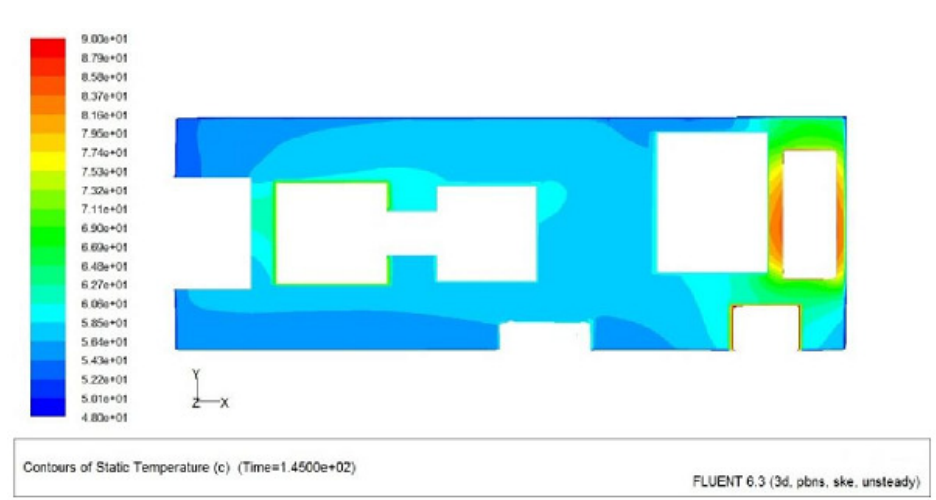

Fig. 6(B). Temperature distribution of the cabin without jet unit of section $\mathrm{z}=2000 \mathrm{~mm}$.

It is easily concluded from the figure that without jet unit, the temperature of most areas within the cabin exceeds the allowable value of $50{ }^{\circ} \mathrm{C}$ and produces a temperature accumulation. The accumulation is especially severe around the gear box and cooling box. Therefore, the jet unit is applied to cool the cabin and enhance the air flow within the cabin so as to achieve the cabin heat balance. 


\section{Jet Units Arrangement and Optimization}

When the cabin reaches heat balance state, according to the conservation of energy, we can get:

$$
Q_{r}+Q_{j}+Q_{e}+Q_{x}=0
$$

For the given equation above, $Q_{r}$ is internal heat source of the cabin, $Q_{j}$ is heat load of the jet unit and which air volume is determined by heat load. Meanwhile, $Q_{e}$ is heat load coming into the cabin and $Q_{x}$ is heat load of fresh air.

$$
Q_{j}=\dot{m} C_{p}\left(T_{W}-T_{c}\right),
$$

where $\dot{m}$ is quality of the cooling gas, $T_{W}$ is delivery air temperature of jet units, $T_{W}=48^{\circ} \mathrm{C} . T_{c}$ is control temperature of the cabin, $T_{c}=50^{\circ} \mathrm{C}$.

The velocity of delivery air is set as $5 \mathrm{~m} / \mathrm{s}$ and the calculated air volume is $6000 \mathrm{~m}^{3} / \mathrm{h}$ to meet the cooling requirements.

\subsection{Arrangement of jet units}

(1) Single jet unit

Heat-dissipating of cooling system accounts for half of the total heat. A single jet unit is arranged above the cooling box to remove heat of cooling box, as shown in Figure 7. The jet unit jets flow from right side to left side to achieve more uniform temperature field, which is calculated through finite volume method.

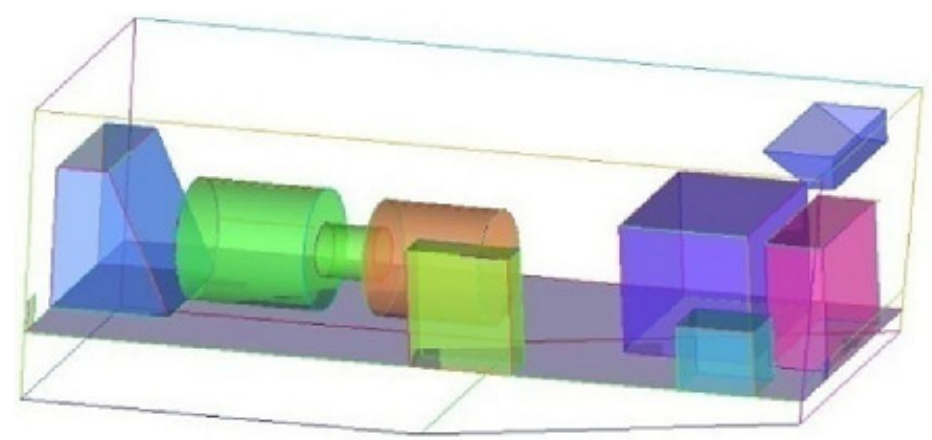

Fig.7. Cabin with a single jet unit.

Figure 8 shows the side view and top view of temperature field. As compared with non-jet unit cabin, jetting with an oblique single jet unit can partly solve the problem of temperature accumulation within the cabin. Nevertheless, there are still some areas, such as the right side of cooling box and surroundings of gear box, where the highest temperature reaches $60{ }^{\circ} \mathrm{C}$ and temperature accumulation occurs. Therefore, to achieve more uniform temperature field, optimizing the structure and layout of the jet unit must be taken into consideration. 


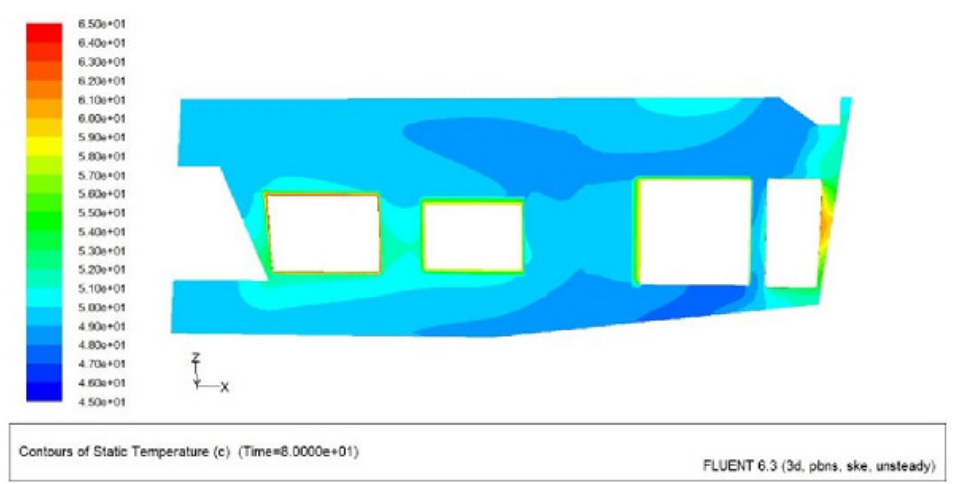

Fig. 8(A). Temperature distribution of single jet unit of section $y=1500 \mathrm{~mm}$.

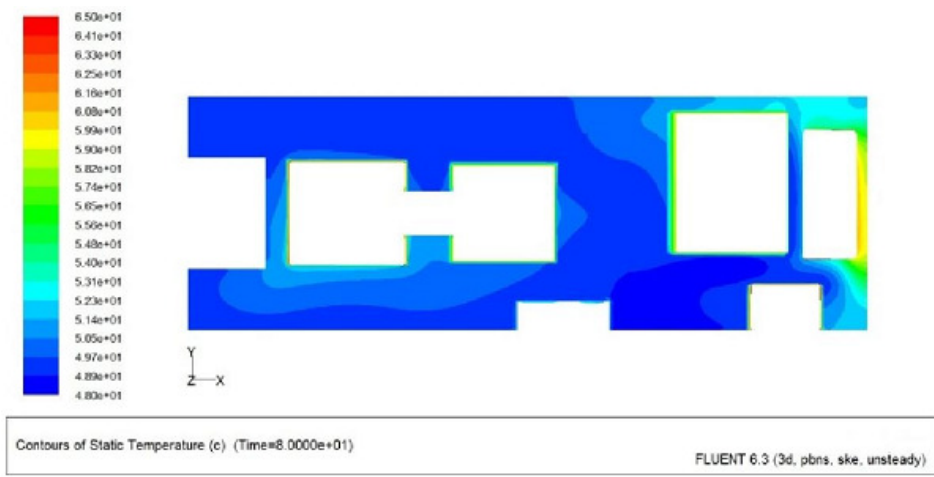

Fig. 8(B). Temperature distribution of single jet unit of section $\mathrm{z}=2000 \mathrm{~mm}$.

(2) Direct and oblique jet of two units

To prevent heat accumulation and obtain a more uniform temperature field around the key components, the jet unit could be split into two portions and placed above the cooling box and gear box with a larger proportion of total heat accumulation. Direct and oblique arrangements of the units are shown in Figures 9 and 10. These two cases are calculated and the results are shown in Figures 11 and 12.

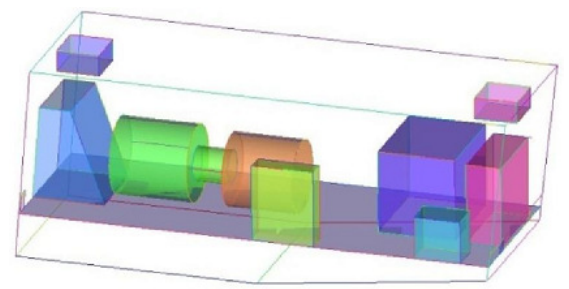

Fig. 9. Arrangement of two units with direct jet

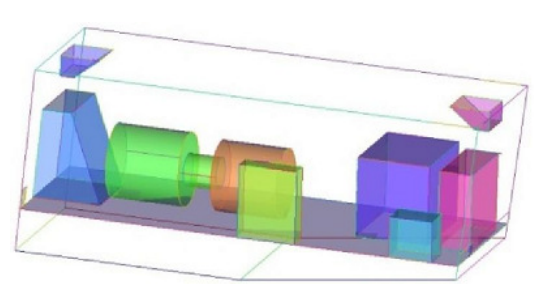

Fig. 10. Arrangement of two units with oblique jet 


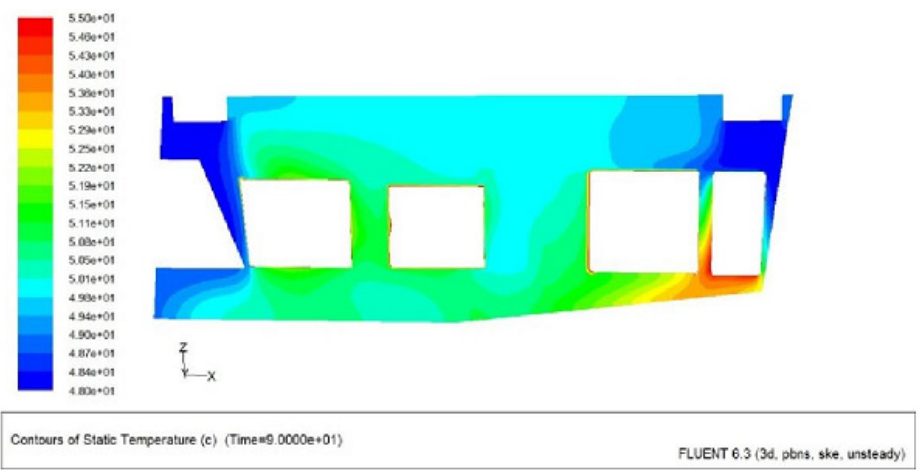

Fig. 11(A). Temperature distribution of two units with direct jet of section $y=1500 \mathrm{~mm}$.

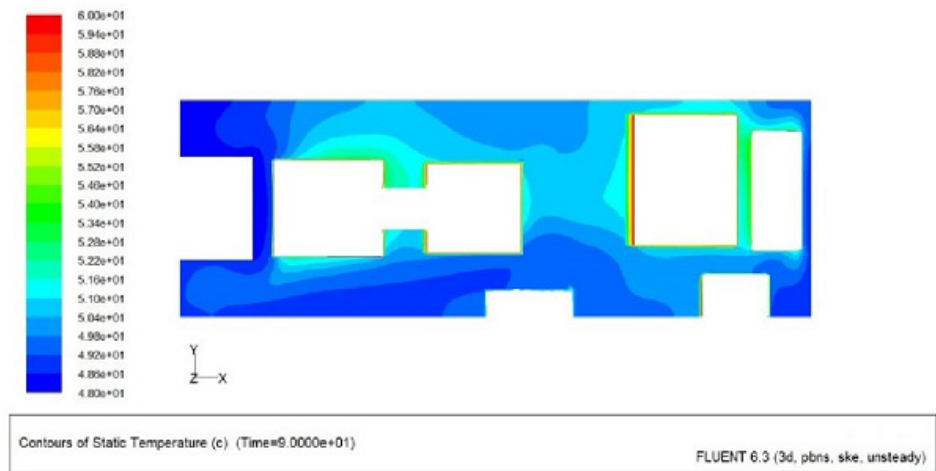

Fig. 11(B). Temperature distribution of two units with direct jet of section $\mathrm{z}=2000 \mathrm{~mm}$.

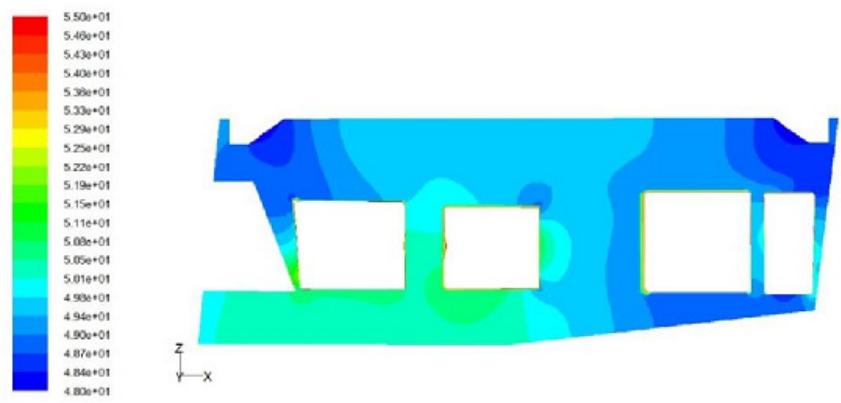

Contours of Static Temperature (c) (Time $=2.2200 \mathrm{e}+02$ ) FLUENT 6.3 (3d, pbns, ske. unsteady)

Fig. 12(A). Temperature distribution of two units with oblique jet of section $y=1500 \mathrm{~mm}$. 


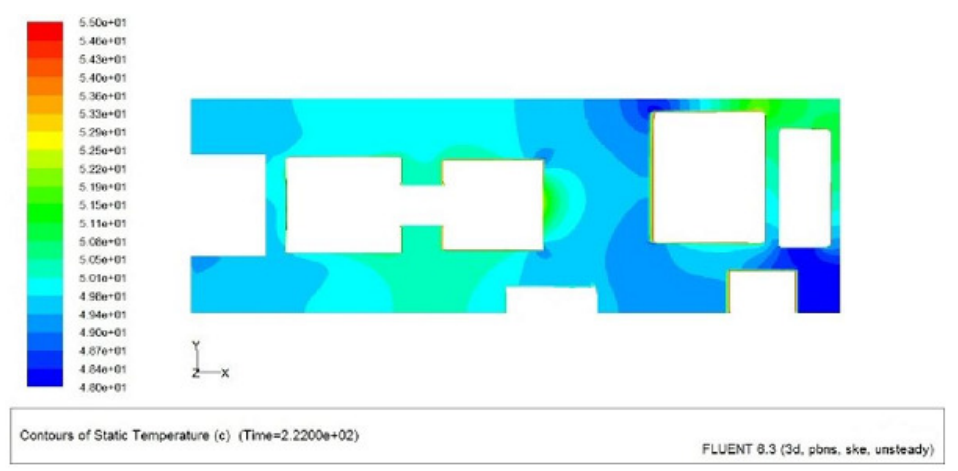

Fig. 12(B). Temperature distribution of two units with oblique jet of section $\mathrm{z}=2000 \mathrm{~mm}$.

Figures 11 and 12 indicate that, in the same jet air flow rate and temperature, the temperature distribution by oblique jet is more uniform than that of the direct jet when the system reaches steady state. The temperature at the bottom of cooling box reaches $55{ }^{\circ} \mathrm{C}$ when direct jet is applied, which may adversely affect component operation in the box. Cold air from jet units has a cooling effect on local region but does not spread over the whole cabin so that temperature gradient throughout the cabin is larger than that with oblique jet. Temperature distribution of the cabin is more uniform using oblique jet and could be mainly controlled below $50{ }^{\circ} \mathrm{C}$ and the temperature gradient is smaller. Oblique jet can increase air turbulence and enhance air diffusions in the cabin which results in more ideal cooling effects.

\subsection{Comparison of different arrangements}

(1) Temperature comparison of control box

Control units in control box are easily damage under high temperature, therefore the requirement of temperature field around the control box is particularly strict (usually should not exceed $50{ }^{\circ} \mathrm{C}$ ). The common line of plane $\mathrm{y}=600 \mathrm{~mm}, \mathrm{z}=1000 \mathrm{~mm}$ or $\mathrm{z}=$ $3000 \mathrm{~mm}$ is selected according to the size of control box. The temperature distribution of each arrangement above and below the control box is shown in Figures 13 and 14.

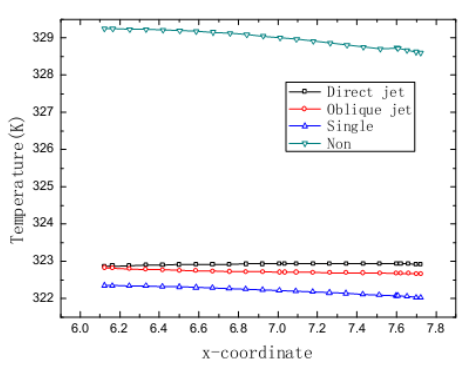

Fig. 13. Temperature distribution above control box

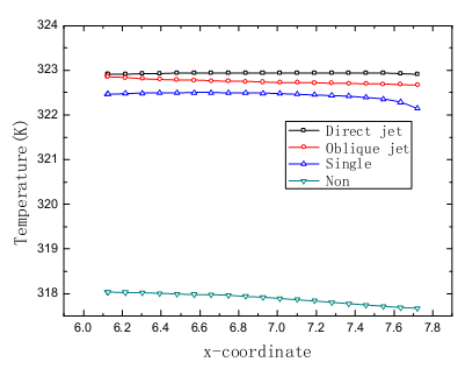

Fig. 14. Temperature distribution below control box 
(2) Temperature comparison of cooling box

Similarly, according to the size of cooling box, common line of plane $y=3600 \mathrm{~mm}, \mathrm{z}=$ $1020 \mathrm{~mm}$ or $\mathrm{z}=3000 \mathrm{~mm}$ is chosen. The temperature distribution above and below the cooling box is shown in Figures 15 and 16.

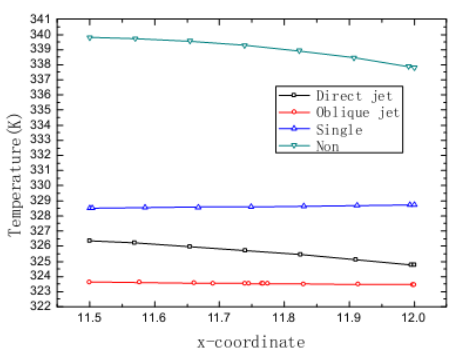

Fig. 15. Temperature distribution above cooling box

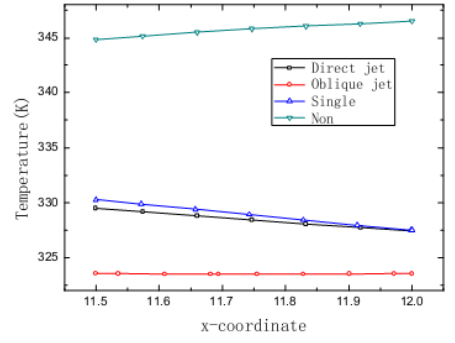

Fig. 16. Temperature distribution below cooling box

From the figures it can be found that, the case for the single-jet unit, two units with direct and oblique jet can all successfully control the temperature to be below $50{ }^{\circ} \mathrm{C}$ $(323 \mathrm{~K})$. In contrast, the temperature of control box for the case without jet unit exceeds $50{ }^{\circ} \mathrm{C}$. Hence, the use of jet unit can meet the requirements and achieve similar results.

For cooling box, the direct jet units have better cooling effect than that of single one. They partially solve the temperature accumulation of cooling box but the ambient temperature cannot fully meet requirements. On the other hand, the oblique jet units provide better cooling effect in controlling the ambient temperature than direct jet units and can make the temperature around the cooling box below $50{ }^{\circ} \mathrm{C}$.

\section{Conclusions}

According to the structure of engine room with $3 \mathrm{MW}$ marine wind turbine, a three-dimensional model is proposed and temperature field inside the cabin is obtained with finite volume method as well as some related heat and flow transfer equations. Jet unit is applied in the cabin to prevent heat from gathering and keep heat balance of the engine room. Conclusions are as follows:

(i) The wind speed, cabin materials, thickness of cabin bulkhead and some other factors have effects on the heat transfer and the coefficient outside the cabin is different along the cabin.

(ii) Under the same jet velocity and air flow temperature, the cooling performance of splitting one jet unit into two parts is better than that of a single unit. The reason is that two jet units blowing in opposite directions can result in turbulence inside the cabin to achieve better heat balance than vertical jet.

(iii) Temperature field analysis of the key components shows that two units blowing in opposite directions can effectively solve heat gathering of all components and meet the requirements of wind turbine system heat balance design. 
(iv) Temperature uniformity of the cabin can be improved by increasing the number of jet units under reasonable layout.

This work completes the heat balance design and maintenance of the $3 \mathrm{MW}$ marine wind turbine, which could be helpful for the optimization of large-capacity wind turbine cooling system.

\section{References}

1. Y. Jiang, W. Yuan, Q. Zhang and Z. Wang, J. NUAA, 40, 199 (2008).

2. Z. Wang, C. Jiang, Q. Ai and C. Wang, R. S. Ener. Rev. 13, 216 (2009).

3. E. C. Morgan, M. Lackner, R. M. Vogel and L. G. Baise, Ener. Conv. Manag. 52, 15 (2011).

4. D. G. Tskhvirashvili, Z. D. Berishvili, V. G. Keshelava, Y. A. Tsagareli, G. S. Mikava and V. K. Topuriya, Pow. Tech. Eng. 24, 23 (1990).

5. W. Yuan and Y. Jiang, World SCI-TECH R\&D, 29, 80 (2007).

6. P. Verboven, N. Scheerlinck, J. de Baerdemaeker and B. M. Nicolaï, J. Food Eng. 43, 41 (2000).

7. H. Shi, Y. Cai and Y. Wang, 2010 Asia-Pacific International Symposium on Aerospace Technology.

8. S. L. Mitchell and T. G. Myers, Int. J. Heat and Mass Transfer, 53, 3540 (2010).

9. F. Mosally, A. S. Wood and A. Al-Fhaid, App. Math. Comp. 130, 87 (2002).

10. F. D. Molina-Aiz, H. Fatnassi, T. Boulard, J. C. Roy and D. L. Valera, Comp. Elec. Agr. 72, 69 (2010).

11. J. Kim, D. Kim and H. Choi, J. Comp. Phys. 171, 132 (2001). 